\title{
Chitosan Reduced Gold Nanoparticles as Novel Carriers for Transmucosal Delivery of Insulin
}

\author{
Devika R. Bhumkar, ${ }^{1}$ Hrushikesh M. Joshi, ${ }^{2}$ Murali Sastry, ${ }^{2,3}$ and Varsha B. Pokharkar ${ }^{1}$
}

Received October 17, 2006; accepted January 30, 2007; published online March 23, 2007

\begin{abstract}
Purpose. Colloidal metallic systems have been recently investigated in the area of nanomedicine. Gold nanoparticles have found themselves useful for diagnostic and drug delivery applications. Herein we have reported a novel method for synthesis of gold nanoparticles using a natural, biocompatible and biodegradable polymer; chitosan. Use of chitosan serves dual purpose by acting as a reducing agent in the synthesis of gold nanoparticles and also promotes the penetration and uptake of peptide hormone insulin across the mucosa. To demonstrate the use of chitosan reduced gold nanoparticles as carriers for drug delivery, we report herein the transmucosal delivery of insulin loaded gold nanoparticles.

Materials and Methods. Gold nanoparticles were prepared using different concentrations of chitosan (from $0.01 \% \mathrm{w} / \mathrm{v}$ up to $1 \% \mathrm{w} / \mathrm{v}$ ). The gold nanoparticles were characterized for surface plasmon band, zeta potential, surface morphology, in vitro diffusion studies and fluorescence spectroscopy. The in vivo studies in diabetic male Wistar rats were carried out using insulin loaded chitosan reduced gold nanoparticles.

Results. Varying concentrations of chitosan used for the synthesis of gold nanoparticles demonstrated that the nanoparticles obtained at higher chitosan concentrations $(>0.1 \% \mathrm{w} / \mathrm{v})$ were stable showing no signs of aggregation. The nanoparticles also showed long term stability in terms of aggregation for about 6 months. Insulin loading of $53 \%$ was obtained and found to be stable after loading. Blood glucose lowering at the end of $2 \mathrm{~h}$ following administration of insulin loaded gold nanoparticles to diabetic rats was found to be 30.41 and $20.27 \%$ for oral (50 IU/kg) and nasal (10 IU/kg), respectively. Serum gold level studies have demonstrated significant improvement in the uptake of chitosan reduced gold nanoparticles.

Conclusions. The synthesis of gold nanoparticles using a biocompatible polymer, chitosan would improve its surface properties for binding of biomolecules. Our studies indicate that oral and nasal administration of insulin loaded chitosan reduced gold nanoparticles has led to improved pharmacodynamic activity. Thus, chitosan reduced gold nanoparticles loaded with insulin prove to be promising in controlling the postprandial hyperglycemia.
\end{abstract}

KEY WORDS: chitosan; gold nanoparticles; insulin; transmucosal.

\section{INTRODUCTION}

The use of nanoparticles in biological applications is being widely explored e g. polymeric nanoparticles, metallic nanoparticles and quantum dots have been found applicable in drug delivery, bioimaging and biosensing (1-3). Nanoparticles have been studied as carriers for oral drug delivery to improve its stability and bioavailability across the GI mucosa (4). Size has been shown to be an extremely important parameter affecting the nanoparticle uptake and cellular internali-

\footnotetext{
${ }^{1}$ Department of Pharmaceutics, Poona College of Pharmacy, Bharati Vidyapeeth University, Pune 411 038, India.

${ }^{2}$ Nanoscience Group, Materials Chemistry Division, National Chemical Laboratory, Pune 411 008, India.

${ }^{3}$ Present address: Tata Chemicals Innovation Centre, Pune 400059 , India.

${ }^{4}$ To whom correspondence should be addressed. (e-mail: vbpokharkar@ yahoo.co.in)
}

ABBREVIATIONS: BGL, blood glucose levels; p.o., peroral; i.n., intranasal; SPB, surface plasmon band. zation. The absolute upper size limit for uptake by intestinal epithelium is $10 \mu \mathrm{m}$ but the amount of uptake certainly increases, as the nanoparticles get smaller (5). The M cells of the Peyer's patches represent a type of lymphatic islands within the intestinal tract and possibly, the major gateway through which particles in the nanoscale range to few micrometers of diameter can be effectively endocytosed (6).

Metal nanoparticles such as gold are excellent candidates for bioconjugation with biologicals (7). Many researchers have shown that biologically active substances with amine functions can bind strongly with gold nanoparticles (8). The tunable shape and size dependant optical properties of gold nanoparticles have been exploited in various surface coatings and biomedical applications (9). They are biocompatible, nontoxic, bind readily to a large range of biomolecules such as amino acids, proteins/enzymes and DNA and expose large surface areas for immobilization of such biomolecules (10-12). The ability to modulate the surface chemistry of gold nanoparticles by binding suitable ligands has important applications in many areas such as novel organic reactions, sensors (both inorganic and biological entities), drug/DNA delivery 
and imaging (13-15). Branched polyethylenimine covalently attached to gold nanoparticles has been investigated for the delivery of plasmid DNA (16). The cytotoxic drug cisplatin was adsorbed on $\mathrm{Au}-\mathrm{Au}_{2} \mathrm{~S}$ nanoparticles via 11-mercaptoundecanoic acid (MUA) layers (17). Gu et al. have shown that gold nanoparticles in toluene react with bis(vancomycin) cystamide in water under vigorous stirring conditions to form vancomycin capped gold nanoparticles; the antibiotic-capped gold nanoparticles showed enhanced antibacterial activity against $E$. coli strains (18). Recently $\mathrm{SiO}_{2}$-core-Au-shell nanoparticles designed to absorb in the near infrared have been used in cancer hyperthermia (19).

Conventional techniques for aqueous synthesis of gold nanoparticles involve Turkevitch process of reduction of $\mathrm{Au}$ (III) $\mathrm{Cl}_{3}$ with trisodium citrate. This process gives uniform and fairly spherical particles but proceeds at a very slow pace, which takes around an hour for complete reduction of $\mathrm{Au}$ (III) $\mathrm{Cl}_{3}$ (20). The two-phase Brust-Schiffrin method usually involves use of organic solvents, which leads to formation of excellent uniform gold nanoparticles. However, the organic solvents render them unsuitable for application in biosensing or delivery of biomolecules (21). Thus, there is a need to develop biocompatible gold nanoparticles for their application in biomolecule delivery. In our previous study we have reported the use of gold nanoparticles synthesized using the reducing agent sodium borohydride for transmucosal delivery of insulin. Also the surface modification of gold nanoparticles was done with aspartic acid for efficient binding of insulin (11).

Oral administration of insulin remains a significant challenge because it is susceptible to hydrolysis and digestion by the acids and enzymes in the gastrointestinal tract. Also bioavailability of insulin is very low due to poor membrane permeability. Low membrane permeability and inadequate stability restricts the potential use of insulin across the transmucosal routes. Protease inhibitors, permeation enhancers, enteric coatings and polymeric microsphere formulations have all been employed towards the development of transmucosal insulin formulations with varying degrees of success (22-25). One possibility to improve the gastrointestinal uptake of perorally poorly absorbed drugs like insulin is their binding to colloidal particles can protect labile molecules from degradation in the gastrointestinal tract and promote the transport of poor-absorbable molecules into systemic circulation. Nasal drug delivery has been investigated as an alternative mode of administering drugs with low bioavailability. The differences in the anatomy and physiological particulars are given Table I.

To improve the stability and uptake of nanoparticles we have reported in the present study, a novel strategy for synthesis and stabilization of gold nanoparticles using a natural, biocompatible polymer, chitosan. Chitosan is well known for its hydrophilic, biocompatible, biodegradable and non-toxic properties $(26,27)$. Use of chitosan nanoparticles for delivery of insulin by oral and nasal route has been reported earlier. Pan et al. reported the formation of positively charged chitosan nanoparticles with the size range of $250-400 \mathrm{~nm}$ by ionotropic gelation method (28). They observed that insulin loaded chitosan nanoparticles at a dose of $21 \mathrm{IU} / \mathrm{Kg}$ after oral administration produced hypoglycemia in alloxan induced diabetic rats. However, Ma et al. have reported an oral dose of $100 \mathrm{IU} / \mathrm{Kg}$ to maintain the serum glucose level at prediabetic levels (29). Chitosan acts as an absorption enhancer and promotes uptake by transient opening of the tight epithelial junctions (30). Hence it was envisaged that use of chitosan along with the metallic carrier would further promote the absorption of a peptide molecule such as insulin. In the present work a novel strategy was undertaken to exploit the use of chitosan as a reducing agent for the production of gold nanoparticles and subsequently load with insulin to demonstrate their use for transmucosal peptide delivery.

\section{MATERIALS AND METHODS}

\section{Materials}

Bovine insulin $\left(\mathrm{C}_{254} \mathrm{H}_{377} \mathrm{~N}_{65} \mathrm{O}_{75} \mathrm{~S}_{6}\right.$, molecular weight 5,800 $\mathrm{Da}$ ) was obtained from Sigma. Chloroauric acid (molecular weight $133, \mathrm{C}_{4} \mathrm{H}_{7} \mathrm{NO}_{4}$ ) was obtained from Aldrich and used

Table I. Comparison of Anatomy and Physiological Parameters of Oral and Nasal Mucosa

\begin{tabular}{|c|c|c|}
\hline Particulars & Oral & Nasal \\
\hline $\mathrm{pH}$ & $\begin{array}{l}\text { Stomach-1-3 } \\
\text { Intestine-5-8 }\end{array}$ & $5.5-6.5$ \\
\hline Surface Area & $200 \mathrm{~m}^{2}$ & $150 \mathrm{~cm}^{2}$ \\
\hline Mucus Layer Thickness & $50-500 \mu \mathrm{m}$ & Respiratory epithelium thickness $100 \mu \mathrm{m}$ \\
\hline Enzyme Composition & $\begin{array}{l}\text { Stomach-pepsins, gastric lipase } \\
\text { Intestine-enteropeptidase, dipeptidase, } \\
\text { glucoamylase, lactase }\end{array}$ & $\begin{array}{l}\text { Carboxylesterase, glutathione, } \\
\text { S-transferase and cytochrome P450 } \\
\text { enzyme isoforms CYP1A, CYP2A, } \\
\text { CYP2E }\end{array}$ \\
\hline Membrane Barrier & $\begin{array}{l}\text { Epithelial cell layer, loose connective } \\
\text { tissue, lamina propria (blood and lymph } \\
\text { vessels, nerves, serous glands, mucus } \\
\text { secretory glands, dense capillaries, } \\
\text { lymphocytes and eosinophils). Tight } \\
\text { junctions- size of the channels less than } \\
10 \AA \text {. Impermeable to molecules with a } \\
\text { radius larger than } 15 \AA \text {. }\end{array}$ & $\begin{array}{l}\text { Divided into: vestibular, respiratory and } \\
\text { olfactory. } \\
\text { Respiratory epithelium-basal, mucus } \\
\text { containing goblet, ciliated, columnar cell } \\
\text { types covered by microvilli. Tight } \\
\text { junctions-pore diameter } 4-8 \AA \text { A. } \\
\text { Impermeable to particles larger than } \\
15-25 \AA \text {. }\end{array}$ \\
\hline
\end{tabular}


as received. Chitosan (degree of deacetylation 90\%, molecular weight determined by viscosity average method by Ubbelhode viscometer was $45,000 \mathrm{Da}$ ) was a gift sample from A \& E Connock Ltd., UK. Alloxan monohydrate was obtained from Spectrochem, Mumbai. The glucose oxidase peroxidase (GOD-POD) kit was purchased from Accurex Biomedicals Pvt. Ltd., Thane, India. ELISA was performed on AxSYM/ Imx Fully Automated Immunoassay System, Abbott, USA and the ELISA kit was obtained from Abbott, USA.

\section{Synthesis of Gold Nanoparticles}

In a typical experiment, $100 \mu \mathrm{l}$ of $1.25 \times 10^{-1} \mathrm{M}$ concentrated aqueous solution of chloroauric acid $\left(\mathrm{HAuCl}_{4}\right)$ was reduced by heating for $15 \mathrm{~min}$ in $100 \mathrm{ml}$ of chitosan solution prepared in $1 \%$ acetic acid to yield a ruby-red solution. The ruby red colored solution yielded an absorbance maximum at $520 \mathrm{~nm}$. Varying chitosan concentrations $(0.05,0.01,0.1,0.2$, 0.5 and $1 \%$ ) were used for reduction of chloroauric acid to determine the effect of chitosan concentration on the formation of gold nanoparticles.

\section{Loading of Insulin onto Gold Nanoparticles}

A calculated amount of insulin was added to dispersion of gold nanoparticles reduced using $0.2 \%$ chitosan ( $\mathrm{pH}$ of blank gold nanoparticles was 5) to yield an insulin concentration of $1.384 \mathrm{IU} / \mathrm{ml}$ in solution (isoelectric point of insulin 5.4). The dispersion was incubated for $16 \mathrm{~h}$ at $2-8^{\circ} \mathrm{C}$ followed by ultracentrifugation at $30,000 \mathrm{rpm}$ for $30 \mathrm{~min}$. The pellet thus obtained was separated from the supernatant solution and redispersed in milli $\mathrm{Q}$ water prior to further characterization. The free insulin present in the supernatant was determined by ELISA and the percentage loading of insulin on the nanoparticles was determined by the following formula:

$$
\begin{aligned}
& \text { \%Loading efficiency } \\
& =\frac{\text { Total amount of insulin added-Amount of insulin in supernatant }}{\text { Total amount of insulin added }} \times 100
\end{aligned}
$$

\section{UV-visible Spectroscopy Measurements}

The change in surface plasmon resonance of gold nanoparticles, before and after loading of insulin, was monitored by UV-Visible spectroscopy measurements, carried out on a Model V-570 Jasco Dual Beam Spectrophotometer.

\section{Transmission Electron Microscopy (TEM) Measurements}

TEM measurements of the gold nanoparticles were performed on a JEOL Model 1200EX instrument operated at an accelerating voltage of $120 \mathrm{kV}$. Samples for TEM analysis were prepared by placing drops of the gold nanoparticle dispersions on carbon-coated TEM copper grids. The mixtures were allowed to dry for $1 \mathrm{~min}$ following which the extra solution was removed using a blotting paper.

\section{Particle Size Analysis}

The particle size analysis was carried out using Gattan software (Pleasanton, CA, USA). The hydrodynamic diameter of gold nanoparticles reduced using $0.2 \%$ w/v chitosan was determined using Mastersizer 2000 Ver. 5.22 (Malvern Instruments, Malvern, UK) based on laser diffractometer. Analysis $(n=3)$ was carried out at room temperature by keeping angle of detection at $90^{\circ}$. Particle size of the sample was measured as such without dilution.

\section{Viscosity Measurements}

The viscosity of gold nanoparticles reduced using varying concentrations of chitosan was measured using Brookfield LVDV III Programmable rheometer (cone-plate viscometer) equipped with Spindle CP-40 (Brookfield Engineering Laboratory, Incorporation, Middleboro). A cone and plate sensor having a diameter of $2.4 \mathrm{~cm}$ with an angle of $0.8^{\circ}$ was used.

\section{In Vitro Diffusion Studies}

Modified Franz diffusion cells were used for in-vitro diffusion studies. The freshly obtained goat nasal membrane was clamped into position between the donor and receiver compartments. The surface area of the membrane exposed to the solution was $3.05 \mathrm{~cm}^{2}$. The diffusion studies were carried out for insulin loaded chitosan reduced gold nanoparticles and control insulin solution. The donor compartment contained appropriate volume of insulin loaded chitosan reduced gold nanoparticles and control insulin solution so that the dose of insulin as $0.55 \mathrm{mg}$. The receiver compartment contained $23 \mathrm{ml}$ of $0.9 \% \mathrm{NaCl}$. The content of the receiver compartment was stirred with the help of a magnetic bead at $100 \mathrm{rpm}$. The membrane was allowed to hydrate for $30 \mathrm{~min}$ prior to experimentation. At specified intervals, $1 \mathrm{ml}$ samples were withdrawn from receiver compartment, and an equivalent amount of $0.9 \% \mathrm{NaCl}$ was added to maintain the constant volume. All experiments were run for $6 \mathrm{~h}$. The samples were assayed for insulin contents by ELISA.

\section{Zeta Potential Measurements}

The surface charge of gold nanoparticles before and after loading of insulin was determined by measurement of zeta potential. The zeta potential was determined using the Zetasizer 300 HAS (Malvern Instruments, Malvern, UK) based on photon correlation spectroscopy. Analysis time was $60 \mathrm{~s}$ and the average zeta potential was determined. The zeta potential of nanoparticulate dispersion was determined as such without dilution.

\section{Fluorescence Spectroscopy Measurements}

Fluorescence spectroscopy was used to study the secondary structure of insulin after binding with chitosan reduced gold nanoparticles. Fluorescence spectra of insulin solution and insulin bound to the gold nanoparticles were recorded on a Schimadzu Spectrofluorometer RF-5301 PC.

\section{In-Vivo Studies}

The insulin loaded gold nanoparticle formulation was tested for blood glucose lowering following oral and nasal administration in diabetic rats. Twelve to thirteen weeks old 


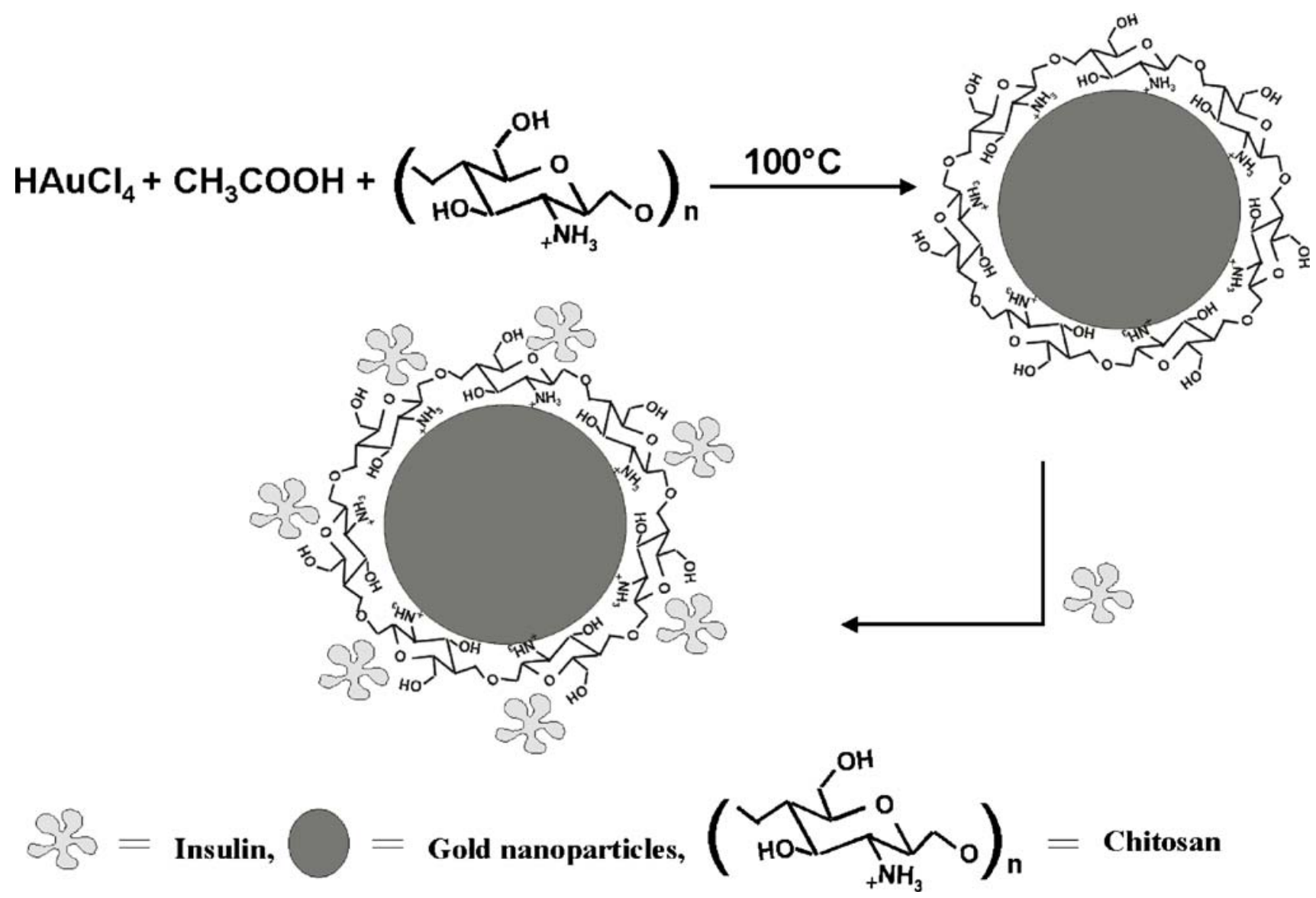

Fig. 1. Schematic diagram showing the capping of chitosan on gold nanoparticles and subsequent loading of insulin on chitosan capped gold nanoparticles.

Wistar male rats weighing 180-200 g each were provided by National Toxicology Centre, Pune, India. The animals were housed under standard conditions of temperature $\left(25^{\circ} \mathrm{C}\right)$, in 12/12 h light and dark cycles and fed with standard pellet diet and water adlibitum. Animal handling was performed according to Good Laboratory Practice (GLP). The Wistar male rats were rendered diabetic prior to the study by intravenous injection of $70-\mathrm{mg} / \mathrm{kg}$ alloxan in sterile normal saline. The animals were divided in groups containing six animals each. They were considered to be diabetic when the baseline glucose levels were above $200 \mathrm{mg} / \mathrm{dl}$.

The formulations administered to the rats were as follows: (1) insulin loaded chitosan reduced gold nanoparticles administered perorally (p.o., $50 \mathrm{IU} / \mathrm{kg}$ ) and intranasally (i.n., $10 \mathrm{IU} /$ $\mathrm{kg}$ ); (2) blank chitosan reduced gold nanoparticles administered p.o. and i.n.; (3) insulin solution administered p.o. (50 IU/kg) and i.n. (10 IU/kg); (4) insulin in $0.2 \%$ chitosan solution (p.o., $50 \mathrm{IU} / \mathrm{kg}$ ) and intranasally (i.n., $10 \mathrm{IU} / \mathrm{kg}$ ).

For intranasal administration, the rats were anaesthetized to prevent sneezing during administration. The animals were anaesthetized using anaesthetic ether by placing them in the anaesthetic chamber. The sedation lasted for about $3 \mathrm{~min}$. The rats were placed in supine position and the samples were delivered using PVC tube connected to a microlitre syringe (31). Blood samples were collected from the retro-orbital plexus of the rats prior to administration of the formulation to establish baseline glucose levels. Similarly, after dosing animals with the various insulin formulations, blood samples were collected at different time intervals over a period of $6 \mathrm{~h}$. Glycemia was determined in the serum samples by the glucose oxidase-peroxidase method and serum insulin levels were determined by ELISA tests. The results shown are the mean values of serum glucose levels and insulin levels of animals in each group (six each). The research proposal for this study was prepared according to Form B of the guidelines of the Committee for the Purpose of Control and Supervision of Experiments on Animals (CPCSEA). The Institutional Animal Ethics Committee (IAEC) of the Poona College of Pharmacy approved the proposal.

\section{Statistical Analysis}

Data was expressed as mean \pm S.D. and statistical analysis was carried out using ANOVA followed by Tukey's test. A level of significance of $P<0.05$ was regarded as statistically significant.

\section{Inductively Coupled Plasma (ICP) Measurements}

Inductively coupled plasma technique was used to determine the gold concentration in the blood serum after administration of the different gold nanoparticle formulations to diabetic rats. Samples were prepared by adding 0.3 $\mathrm{ml}$ of $35 \% 36 \mathrm{~N} \mathrm{HCl}, 0.1 \mathrm{ml} 55 \%$ of $12 \mathrm{~N} \mathrm{HNO}_{3}$ to weighed 


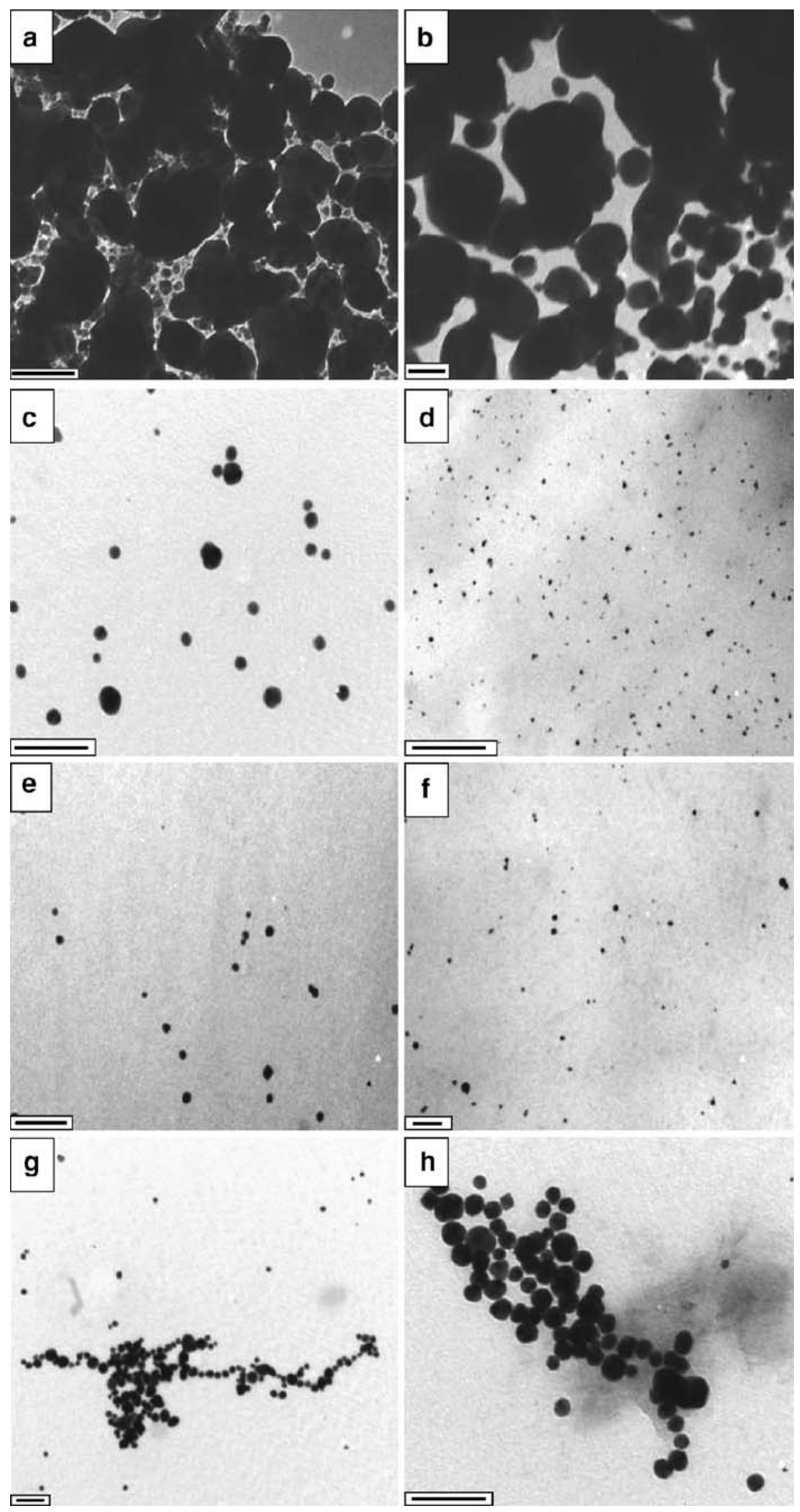

Fig. 2. TEM images of blank gold nanoparticles reduced using varying concentrations of chitosan a $0.01 \% \mathrm{w} / \mathrm{v}, \mathbf{b} 0.05 \% \mathrm{w} / \mathrm{v}$, c $0.1 \% \mathrm{w} / \mathrm{v}, \mathbf{d}$ $0.2 \% \mathrm{w} / \mathrm{v}, \mathbf{e} 0.5 \% \mathrm{w} / \mathrm{v}$ and $\mathbf{f} 1 \% \mathrm{w} / \mathrm{v}$, and insulin loaded gold nanoparticles reduced using $0.2 \% \mathrm{w} / \mathrm{v}$ chitosan $\mathbf{g}$ and $\mathbf{h}$. 

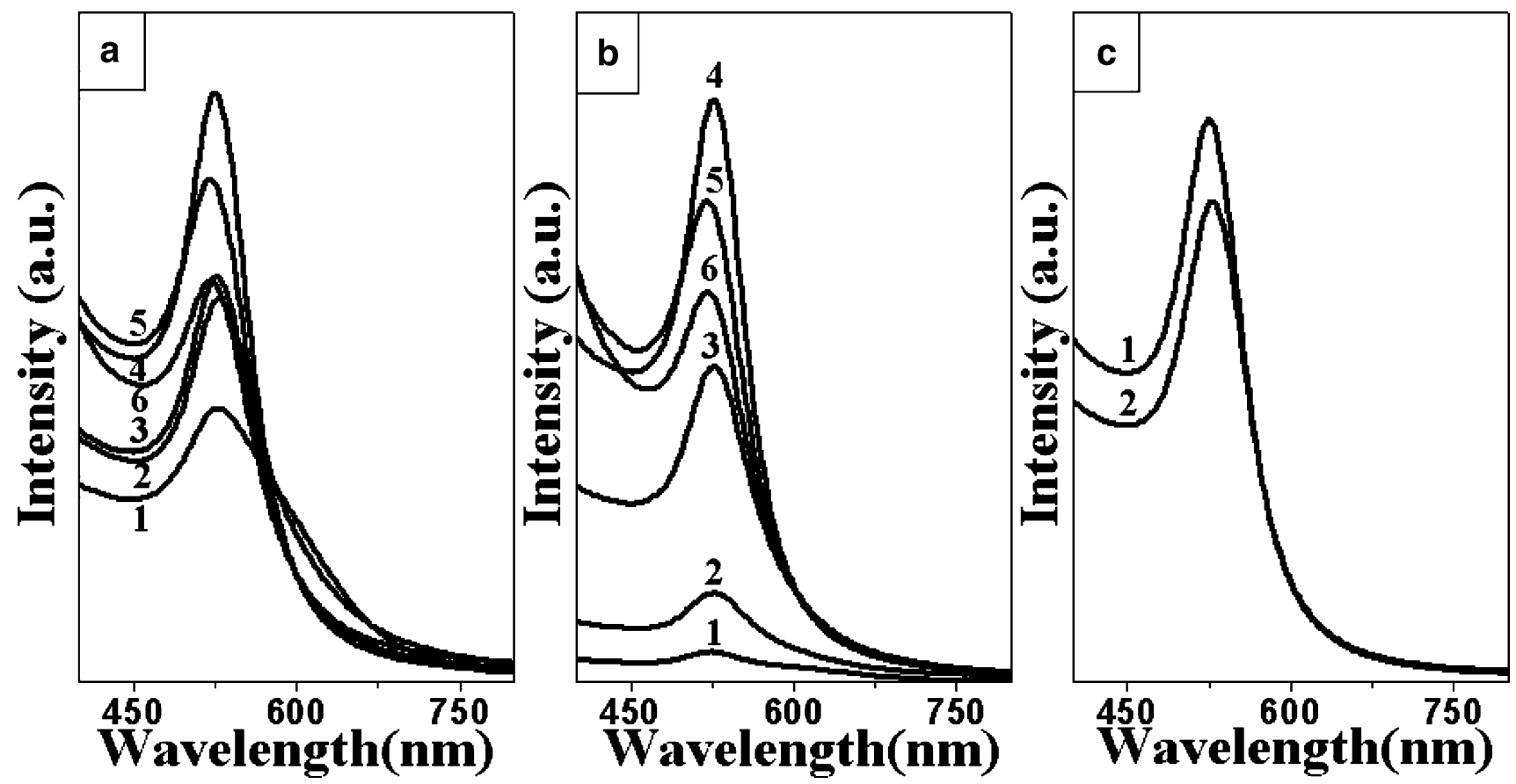

Fig. 3. Visible absorption spectra of gold nanoparticles synthesized using varying concentrations of chitosan a $t=0$; $\mathbf{b} t=6$ months, where curve (1) $0.01 \% \mathrm{w} / \mathrm{v}$, (2) $0.05 \% \mathrm{w} / \mathrm{v}$, (3) $0.1 \% \mathrm{w} / \mathrm{v}$, (4) $0.2 \% \mathrm{w} / \mathrm{v},(5) 0.5 \% \mathrm{w} / \mathrm{v}$, (6) $1 \% \mathrm{w} / \mathrm{v}$. c Visible absorption spectra recorded from the chitosan $(0.2 \% \mathrm{w} / \mathrm{v})$ reduced gold nanoparticle dispersion before (curve 1) and after loading with insulin (curve 2).

amount of blood serum and diluting it up to $2 \mathrm{ml}$. Gold concentration in the above samples were determined on ICP OES Perkin Elmer Optima 3000 instrument.

\section{RESULTS AND DISCUSSION}

Chitosan is known to act as a penetration enhancer for proteins and vaccines administered across the mucosal routes (32). In the present study chitosan was used considering its biocompatibility, chemical nature and absorption enhancement properties. Owing to its electronegative property, chitosan can be used as a reducing agent to obtain the gold nanoparticles, and being a polyelectrolyte it would also act as an electrostatic stabilizer. It was envisaged that this strategy would render dual advantage by providing sufficient charge through the amino groups (Fig. 1), which will aid in the subsequent attachment of the biomolecules as well render optimum stability and subsequently help to improve the uptake of the nanoparticles. Varying chitosan solution concentrations used for the formation of gold nanoparticles demonstrated that lower concentrations of $0.01 \% \mathrm{w} / \mathrm{v}-0.05 \%$ $\mathrm{w} / \mathrm{v}$ resulted in aggregated particles (Fig. 2). However, concentrations of $0.1 \% \mathrm{w} / \mathrm{v}$ and above showed no aggregation. This was reflected in the surface plasmon band (SPB) of the nanoparticles, which showed shift to 528 and $530 \mathrm{~nm}$ with $0.01 \% \mathrm{w} / \mathrm{v}$ chitosan and $0.05 \% \mathrm{w} / \mathrm{v}$ chitosan, respectively, (Fig. 3a). Gold nanoparticles exhibits SPB at $520 \mathrm{~nm}$ due to collective oscillations of the electron at the surface of the nanoparticles (6s electrons of the conduction band for gold nanoparticles) that is correlated with the electromagnetic field of the incoming light, i.e. the excitation of the coherent oscillation of the conduction band. Noble metals show different optical properties at nanoscale than its bulk counter part. These properties are dependent on composition, size, shape and surrounding medium of the particles. SPB of gold nanoparticles comes in the visible region. It can be used to monitor shape, size and aggregation of the nanoparticles. The surface plasmon band is sensitive to interparticle distance due to overlap of dipole resonance from the neighboring nanoparticles. To elucidate the cause of aggregation at lower chitosan concentrations, zeta potential measurements were carried out. The measurement of zeta potential allows predictions about the stability of colloidal aqueous dispersions. Usually, particle aggregation is less likely to occur for charged particles with optimum zeta potential $(\varsigma>30 \mathrm{mV})$ due to electrostatic repulsions. The zeta potential and standard deviation values of gold nanoparticles before and after loading of insulin are shown in Table II. The zeta potential measurement revealed that at lower concentrations

Table II. Zeta Potential of Blank Gold Nanoparticles Synthesized Using Varying Concentrations of Chitosan and Insulin Loaded Chitosan Reduced Gold Nanoparticles

Gold Nanoparticles Reduced

with Different Chitosan Concentrations

Zeta Potential $(\mathrm{mV})$

\begin{tabular}{lc}
\hline $0.01 \%$ & +4.23 \\
$0.05 \%$ & +7.02 \\
$0.1 \%$ & +58.3 \\
$0.2 \%$ & +62.7 \\
$0.5 \%$ & +51.3 \\
$1 \%$ & +50.2 \\
Insulin loaded gold nanoparticles & +65.2
\end{tabular}

reduced with $0.2 \%$ chitosan 


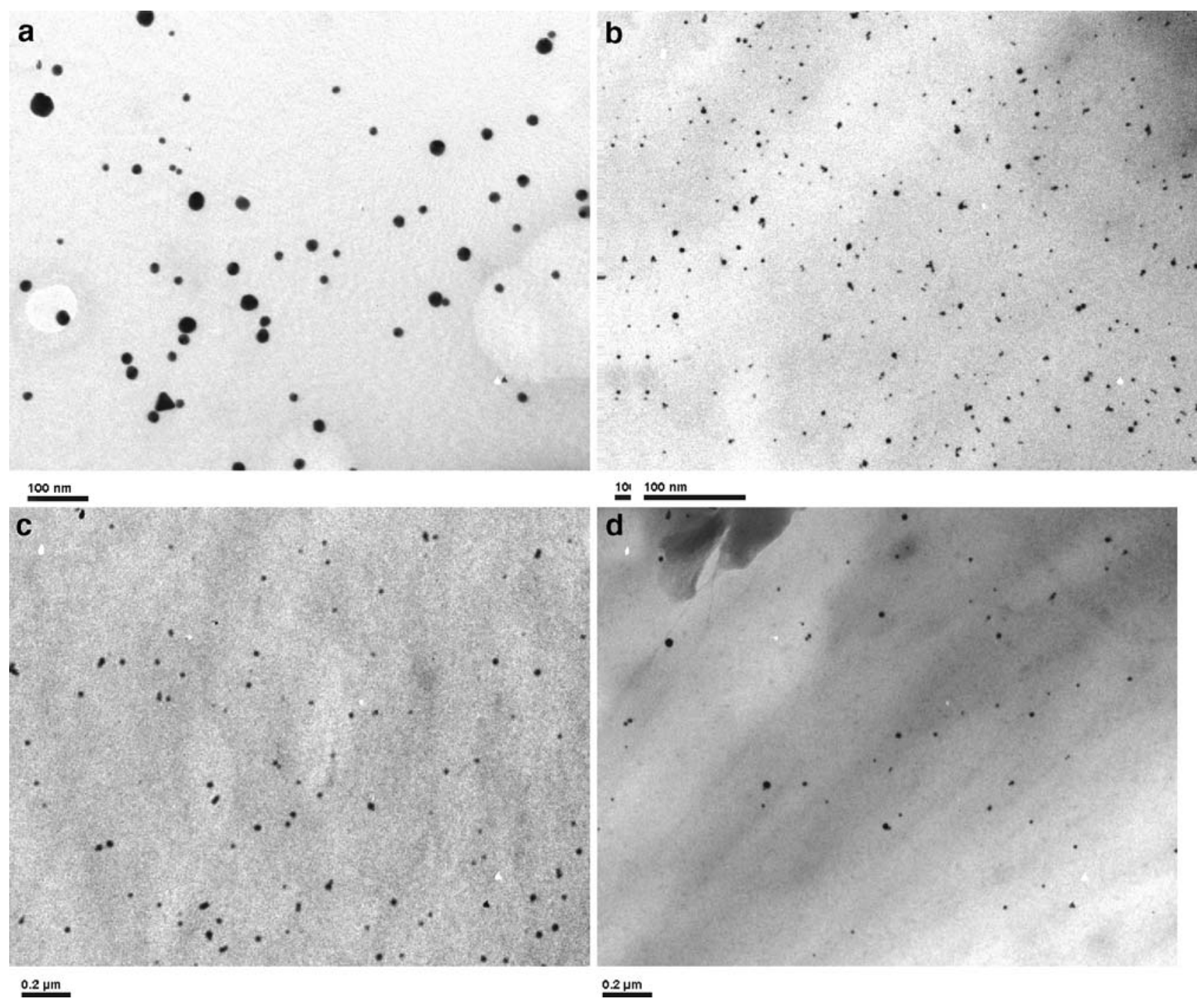

Fig. 4. TEM images after 6 months of blank gold nanoparticles reduced using varying concentrations of chitosan a $0.1 \%$ w/v, b $0.2 \%$ w/v, c $0.5 \% \mathrm{w} / \mathrm{v}, \mathbf{d} 1 \% \mathrm{w} / \mathrm{v}$.

$(0.01 \% \mathrm{w} / \mathrm{v}$ and $0.05 \% \mathrm{w} / \mathrm{v})$ of chitosan the values were below $+10 \mathrm{mV}$. It is well documented that the sodium borohydride reduced gold nanoparticles are negatively charged (8). However, reversal of the sign (from negative to positive) was observed for the chitosan reduced gold nanoparticles. This indicated that the coating/ wrapping of the gold nanoparticles with the cationic chitosan results in the

Table III. Viscosity of Blank Gold Nanoparticles Synthesized Using Varying Concentrations of Chitosan

Chitosan Concentration (\%w/v) Viscosity (Pa s) at Stress $10.21 \mathrm{~Pa}$

\begin{tabular}{ll}
\hline 0.01 & 0.0017 \\
0.05 & 0.0016 \\
0.1 & 0.0017 \\
0.2 & 0.0020 \\
0.5 & 0.0033 \\
1 & 0.0034 \\
\hline
\end{tabular}

positively charged nanoparticles. At the lower concentration the charge rendered is insufficient and leads to sensitization of the particles causing aggregation, also evidenced in the TEM images (Fig. 2) (33). However, at higher concentration $(>0.1 \% \mathrm{w} / \mathrm{v})$ sufficient charge $(+58.3 \mathrm{mV})$ is rendered making them electrostatically stable. The effect of molecular weight of polysaccharide plays an important role in its disposition around the nanoparticles. Bertholon et al. have studied the conformation of polysaccharide chains grafted on nanoparticle surface as a function of method of polymerization by electronic paramagnetic resonance spectroscopy (34). Their studies revealed a folded conformation with very high molecular weight polysaccharide prepared by redox radical emulsion polymerization method. In our study, we have obtained metallic nanoparticles using chitosan as a reducing agent. Chitosan molecular weight was 45,000 Da. Hecne, based on the earlier report of Bertholon et al. we hypothetise that the chitosan might be present in an unfolded conformation on the gold nanoparticle surface. The stability of nanoparticles monitored over six months showed that gold 

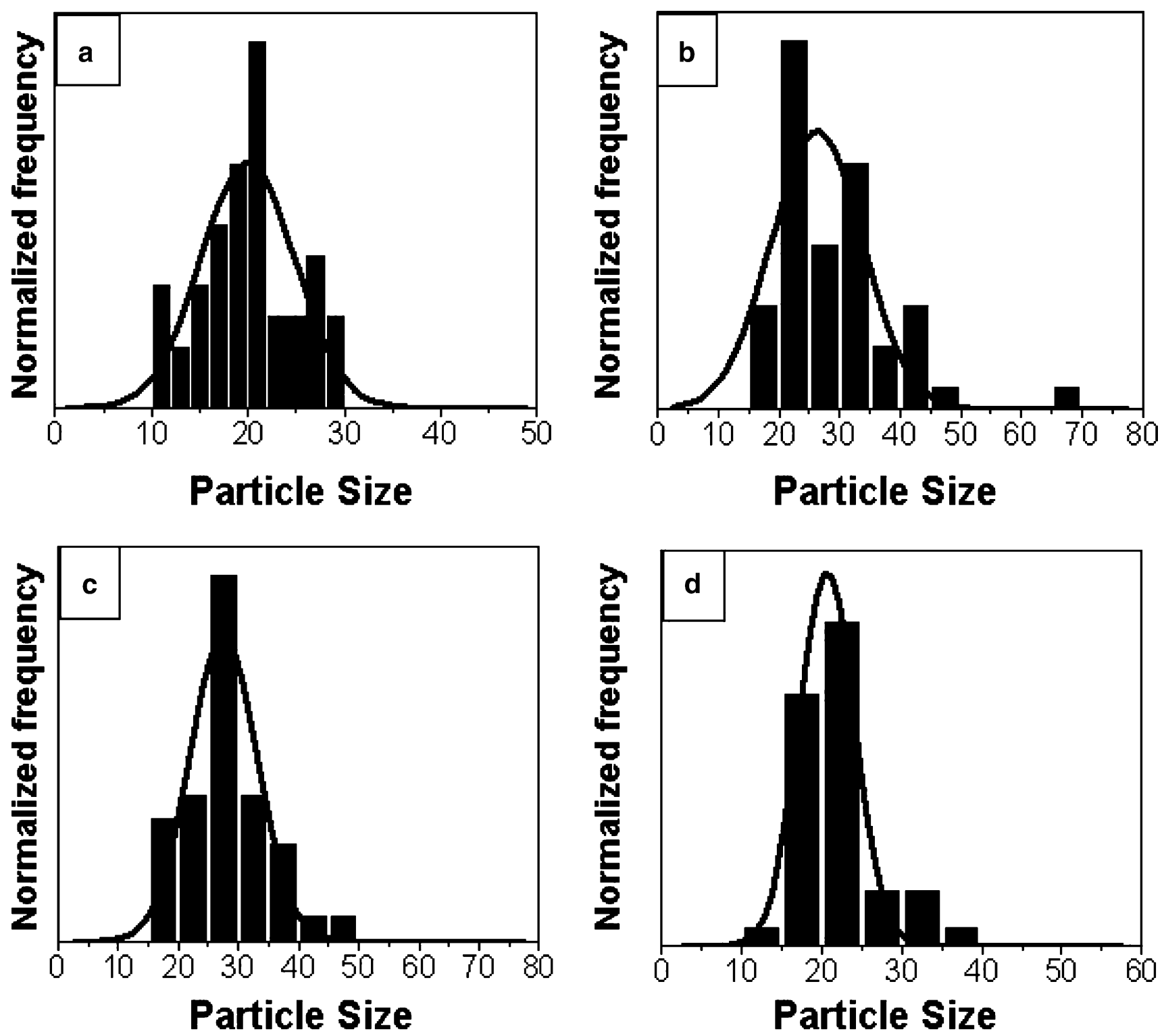

Fig. 5. Particle size distribution of gold nanoparticles synthesized using varying concentrations of chitosan a $0.1 \%$ w/v b $0.2 \%$ w/v c $0.5 \%$ w/v d $1 \% \mathrm{w} / \mathrm{v}$.

nanoparticles synthesized using higher concentrations of chitosan $(0.1 \% \mathrm{w} / \mathrm{v}-1 \% \mathrm{w} / \mathrm{v})$ had no shift in SPB (Fig. 3b), and showed no aggregation (Fig. 4), indicating that nanoparticles formed were stable. Thus, the long-term stability at higher concentration could be attributed to the electrostatic and mechanical barrier properties of chitosan. The viscosity of gold nanoparticles reduced using varying concentrations of chitosan was determined. It was observed that the viscosity was higher at $0.5 \% \mathrm{w} / \mathrm{v}$ chitosan (Table III). Taking into consideration the stability and viscosity of the gold nanoparticles, nanoparticles synthesized using $0.2 \% \mathrm{w} / \mathrm{v}$ chitosan were chosen for subsequent loading of insulin.

Curves 1 and 2 in Fig. 3c correspond to UV-Vis absorption spectra recorded from the $0.2 \% \mathrm{w} / \mathrm{v}$ chitosan reduced gold nanoparticle solution before and after loading of insulin, respectively. In case of blank nanoparticles a prominent absorption at $520 \mathrm{~nm}$ was observed due to the excitation of surface plasmon vibrations in the gold nanoparticles. After loading of nanoparticles with insulin, the SPB shifted to $528 \mathrm{~nm}$. In both the cases $(0.2 \% \mathrm{w} / \mathrm{v}$ chitosan reduced blank gold nanoparticles and insulin loaded gold nanoparticles) broadening of the SP band was not evidenced suggesting no aggregation of nanoparticles. The TEM images recorded from blank nanoparticles $(0.2 \% \mathrm{w} / \mathrm{v}$ chitosan, Fig. $2 \mathrm{~d})$ and insulin loaded nanoparticles (Fig. $2 \mathrm{~g}$ and $\mathrm{h}$ ) indicated that the over all assemblies of the nanoparticles were not affected and so the shift in band after exposure to insulin may be attributed to surface complexation of insulin and not to aggregation of nanoparticles. The particle size range of the nanoparticles was between 10-50 nm as shown in Fig. 5. No significant effect on the particle size distribution of gold nanoparticles was observed with varying concentration of chitosan. Insulin loading on the nanoparticles also did not lead to any effect on the particle size. The particle size determined using photon 


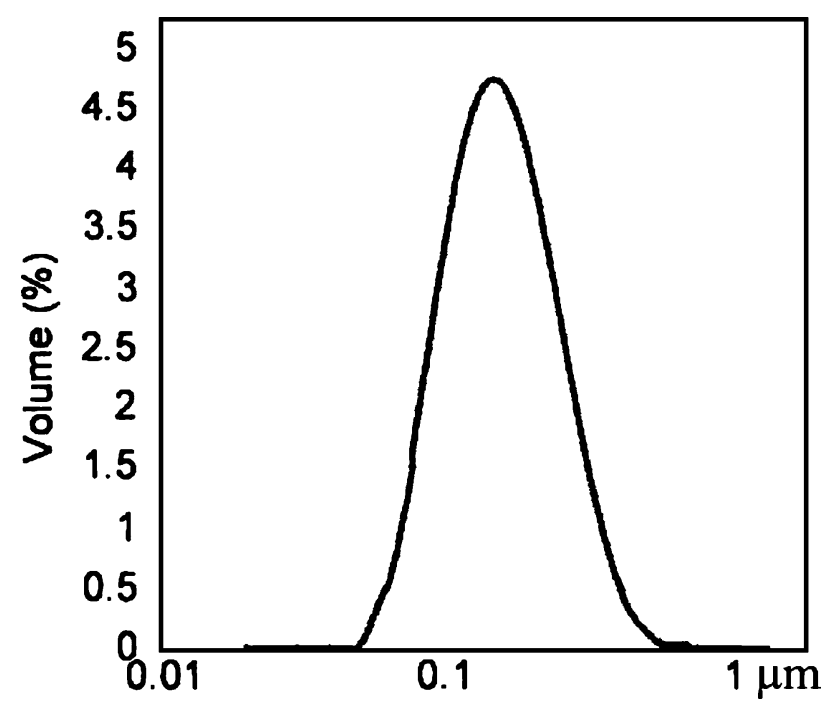

Fig. 6. Hydrodynamic diameter of gold nanoparticles synthesized using $0.2 \% \mathrm{w} / \mathrm{v}$ chitosan.

correlation spectroscopy revealed a monodisperse nature of the $0.2 \% \mathrm{w} / \mathrm{v}$ chitosan reduced gold nanoparticles (Fig. 6). The hydrodynamic diameter of $0.2 \%$ chitosan reduced gold nanoparticles was found to be $100 \mathrm{~nm}$.

The loading efficiency of insulin on chitosan reduced gold nanoparticles was found to be $53 \%$. The nature of binding of insulin to the gold nanoparticles plays vital role in the release and subsequent activity. Reduction of chloroauric acid with chitosan results in formation of chitosan coated gold nanoparticles. The charge on these blank chitosan reduced gold nanoparticles was found to be positive (Table II). The $\mathrm{pH}$ of blank gold nanoparticles was found to be 5 and the isoelectric point of insulin is 5.4. Thus the binding of insulin to the chitosan reduced gold nanoparticles would be predominantly through hydrogen bonding. The stability of insulin after binding with gold nanoparticles was studied using fluorescence spectroscopy. Insulin solution and insulin loaded chitosan

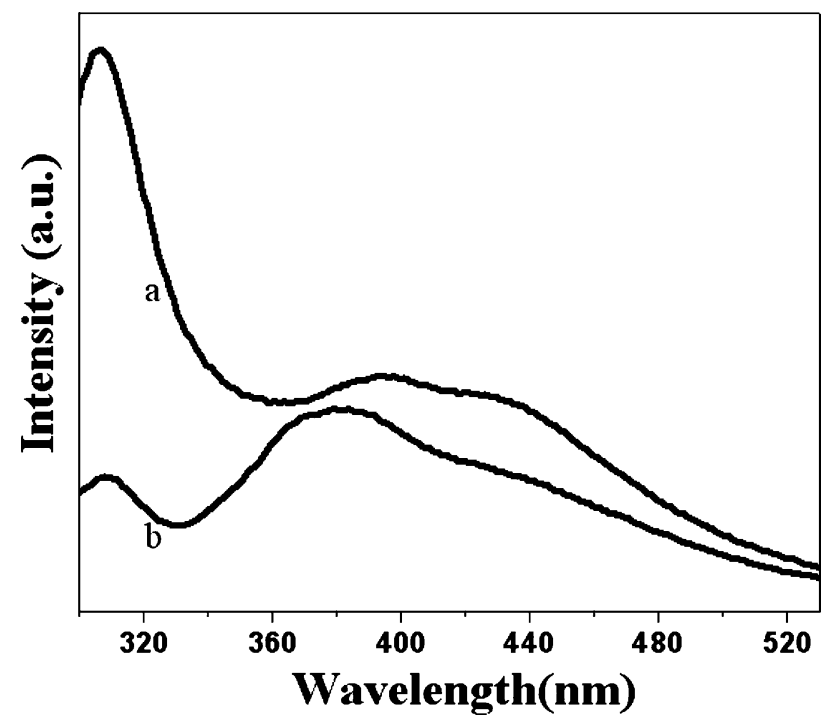

Fig. 7. Fluorescence spectra of a insulin solution, $\mathbf{b}$ insulin loaded chitosan reduced gold nanoparticles.

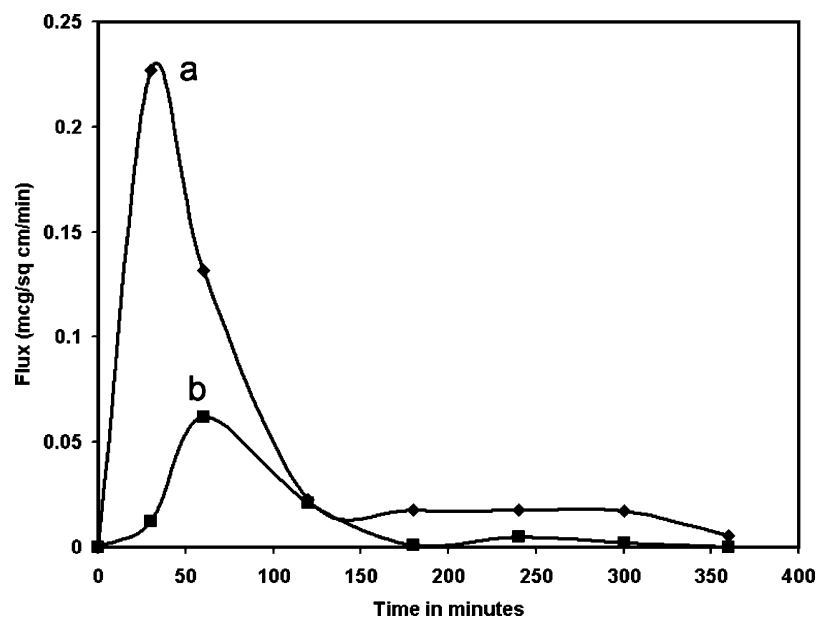

Fig. 8. In vitro diffusion study of a insulin loaded $0.2 \% \mathrm{w} / \mathrm{v}$ chitosan reduced gold nanoparticles, $\mathbf{b}$ free insulin.

reduced gold nanoparticles were excited at $280 \mathrm{~nm}$. The fluorescence emission spectra observed at $305 \mathrm{~nm}$ (Fig. 7) indicated that the side chains of tryptophan have not been exposed and that the secondary structure of insulin is not perturbed after complexation with gold nanoparticles. The in vitro diffusion studies were carried out to determine the permeation of insulin loaded gold nanoparticles across the nasal mucosa and compare it with free insulin. It was observed that the insulin loaded gold nanoparticles showed 4-5 times greater permeation as compared to free insulin (Fig. 8). The difference in the flux obtained could be attributed to the difference in the permeability. This is in agreement with the results reported by Cremaschi et al. where they found that permeability of insulin coated nanobeads up to $490 \mathrm{~nm}$ size had 3.4 times higher flux as compared to free insulin (35).

The pharmacodynamic efficiency of insulin loaded gold nanoparticles was evaluated by monitoring blood glucose levels (BGL) in diabetic rats. The mean serum glucose levels determined in samples from diabetic rats collected before

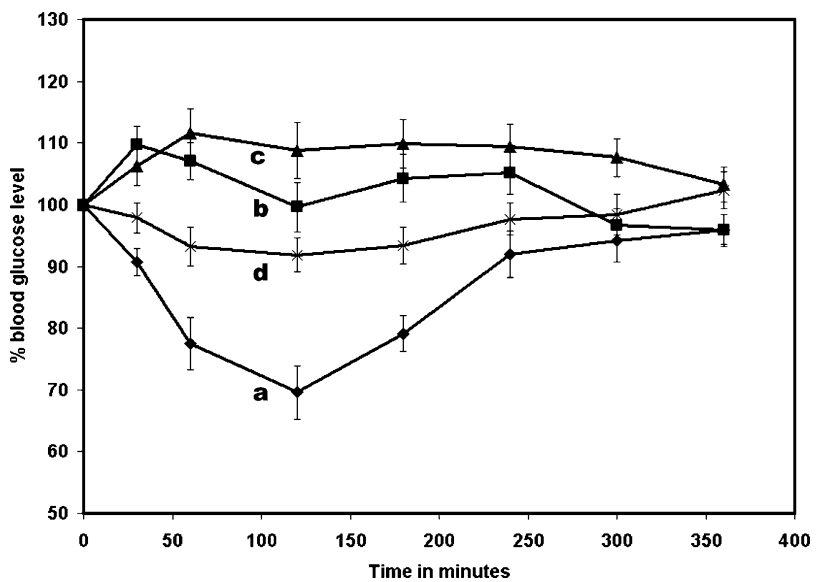

Fig. 9. Percent reduction in blood glucose level after oral administration of a insulin loaded chitosan reduced gold nanoparticles, b blank chitosan reduced nanoparticles, $\mathbf{c}$ insulin solution, $\mathbf{d}$ insulin in chitosan solution (Dose $50 \mathrm{IU} / \mathrm{Kg}, n=6$ ). 


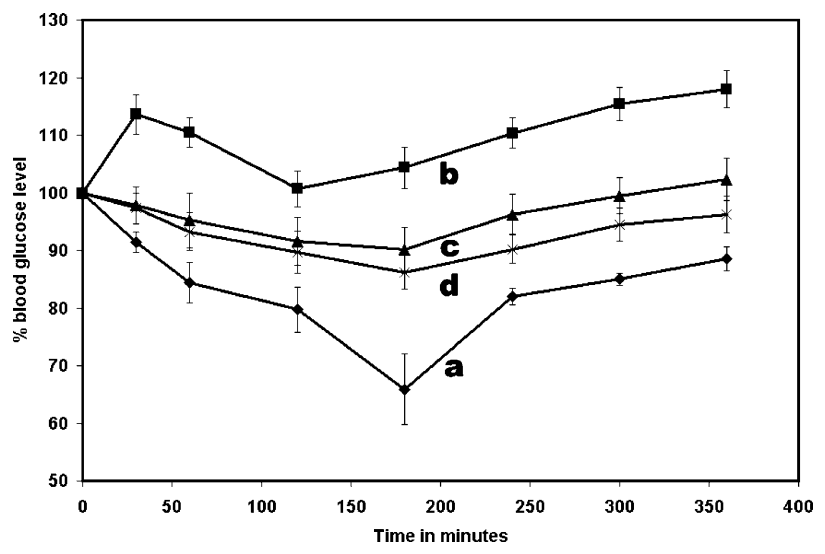

Fig. 10. Percent reduction in blood glucose level after nasal administration of a insulin loaded chitosan reduced gold nanoparticles, b blank chitosan reduced nanoparticles, $\mathbf{c}$ insulin solution, d insulin in chitosan solution (Dose $10 \mathrm{IU} / \mathrm{Kg}, n=6$ ).

administration of the insulin-gold nanoparticle formulations were taken as the baseline values. The percentage BGL relative to this baseline (taken as $100 \%$ ) at each time interval after dosing the diabetic rats with the different insulin formulations was measured. The mean percentage BGL versus time profile obtained after subcutaneous administration of insulin serves as a reference for subsequent studies using the nanoparticle based insulin formulations. As reported in our earlier studies, subcutaneous administration of insulin (dose $5 \mathrm{IU} / \mathrm{kg}$ ) lead to a rapid decrease $(53 \%)$ in BGL in diabetic rats within $2 \mathrm{~h}$ post administration (11). Insulin absorption via the subcutaneous route is sustained and, thus, does not mimic the normal pulsatile pattern of endogenous insulin secretion. After a meal, the injection regimen should aim to provide peak post-prandial
Table IV. Serum Gold Levels in ppm after Oral and Nasal Administration of Insulin Loaded Chitosan Reduced Gold Nanoparticles $(n=6)$

\begin{tabular}{ccc}
\hline \multirow{2}{*}{ Time(min) } & \multicolumn{2}{c}{ Serum Gold Levels in ppm } \\
\cline { 2 - 3 } & Oral & Nasal \\
\hline 120 & $0.059 \pm 0.01$ & $0.061 \pm 0.01$ \\
240 & $0.072 \pm 0.02$ & $0.056 \pm 0.01$ \\
360 & $0.063 \pm 0.02$ & $0.053 \pm 0.03$ \\
\hline
\end{tabular}

blood concentrations of insulin to match the post-prandial hyperglycemia. However, due to the sustained absorption, insulin concentrations between meals may be inappropriately high which commonly results in episodes of hypoglycemia. Subcutaneous or intramuscular injections of insulin results in peripheral hyperinsulinemia, resulting in the exacerbation of the macrovascular complications of diabetes $(36,37)$. Due to these difficulties in achieving a normal physiological profile of insulin by injectable therapy, transmucosal delivery of insulin using gold nanoparticles offers a promising gateway.

The time dependent variation in BGL following the administration of different insulin formulations perorally (p.o) and intranasally (i.n.) to diabetic rats is illustrated in Figs. 9 and 10, respectively. In some of the experiments, a small increase in BGL above the baseline is observed. This increase may be attributed to metabolic changes and endogenous secretion of glucagon in the diabetic rats due to stress in the animals during blood sampling $(38,39)$. In both the p.o. (Fig. 9) and i.n. (Fig. 10) administration protocols, we observed that blank chitosan reduced gold nanoparticles did not result in lowering of BGL in the diabetic rats. Oral

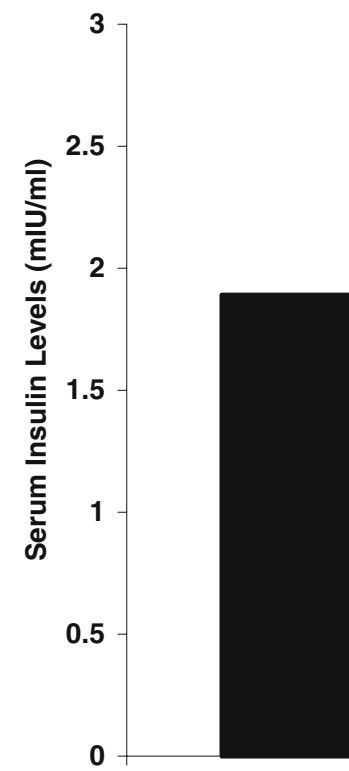

A

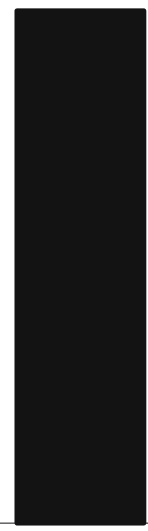

B

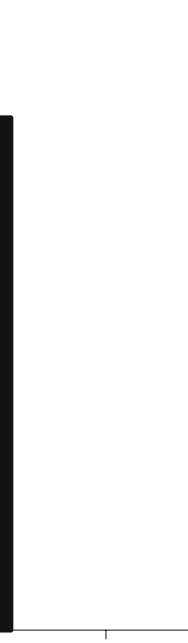

Formulations

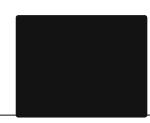

C

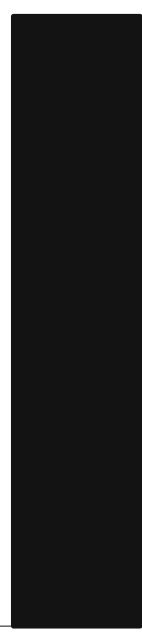

$E$

Fig. 11. Serum insulin levels at the end of $2 \mathrm{~h}$ after $A$ oral administration of insulin loaded on chitosan reduced gold nanoparticles (50 IU/kg), $B$ nasal administration of insulin loaded on chitosan reduced gold nanoparticles (10 IU $/ \mathrm{kg}), C$ insulin solution administered orally $(50 \mathrm{IU} / \mathrm{kg}$ ), $D$ insulin solution administered nasally (10 IU/kg), $E$ subcutaneous administration of insulin solution $(5 \mathrm{IU} / \mathrm{kg})(n=6)$. 
administration of insulin solution (Fig. 9) did not result in a detectable reduction in BGL even after $6 \mathrm{~h}$ of dosage, mainly attributed to poor uptake and enzymatic degradation of insulin in the gastrointestinal tract. In contrast, the nasal administration of insulin solution did lead to $10 \%$ reduction in BGL. However, we found that oral administration of insulin loaded chitosan reduced gold nanoparticles resulted in significantly lowering of BGL up to $30.41 \%$ as compared with insulin solution $(P<0.01)$, clarifying the ability of the nanoparticles to make the loaded protein more stable and protect it from the harsh environment of the gastrointestinal tract. This drop was comparatively higher than our earlier reported results where $18 \%$ reduction was obtained after oral administration of insulin loaded sodium borohydride reduced gold nanoparticles (11). Thus, the reduction in BGL post administration of insulin loaded chitosan reduced gold nanoparticles was found to be significantly higher than sodium borohydride reduced gold nanoparticles. The maximum reduction in BGL was at $120 \mathrm{~min}$ after administration of chitosan reduced gold nanoparticles, which was earlier than our reported results of insulin loaded sodium borohydride reduced gold nanoparticles (180 min). This shows that oral absorption of gold nanoparticles is enhanced after its surface modification with chitosan. This behavior of chitosan reduced gold nanoparticles could demonstrate the role of chitosan as a mucoadhesive polymer causing the nanoparticles to adhere the biomembrane. This facilitates the interaction of chitosan with cell membrane resulting in a structural reorganization of the tight junctions, followed by enhanced transport of associated insulin through the paracellular pathway. Pan et al. have proved the greater efficiency of insulin loaded chitosan nanoparticles compared to the aqueous solution of chitosan in reducing the BGL in diabetic Wistar rats (28). Lowman et al. have reported the use of mucoadhesive hydrogels of poly (methacrylic-g-ethylene glycol) for oral delivery of insulin $(25 \mathrm{IU} / \mathrm{kg}$ ) where $20 \%$ reduction in BGL was obtained at $2 \mathrm{~h} \mathrm{(39).} \mathrm{Thus,} \mathrm{our} \mathrm{approach} \mathrm{to} \mathrm{make} \mathrm{insulin}$ administration easier, more efficient and to make more stable nanoparticle was successful. Nasal administration of blank chitosan reduced gold nanoparticles did not result in reduction in BGL. A significant reduction in BGL following nasal administration of insulin loaded chitosan reduced gold nanoparticles was found as compared to insulin solution $(P<0.01)$. At relatively lower dose of $10 \mathrm{IU} / \mathrm{kg}$, nasal administration lead to 20.27 and $34.12 \%$ reduction at the end of 2 and $3 \mathrm{~h}$, respectively. Thus, comparing it with oral administration (50 IU/kg), the nasal administration (10 IU/kg) has shown much interesting results. Our earlier work with sodium borohydride reduced gold nanoparticles demonstrated that nasal administration of the same at $20 \mathrm{IU} / \mathrm{Kg}$ resulted in $50 \%$ reduction of BGL at the end of $3 \mathrm{~h}$ (11). The higher reduction in BGL following nasal administration of gold nanoparticles as compared to oral is attributed due to the differences in the anatomy and physiological particularities as depicted in Table I (40-45). The serum insulin levels determined at the end of the $2 \mathrm{~h}$ were consistently higher for chitosan reduced gold nanoparticles as compared to insulin solution when administered by oral and nasal routes (Fig. 11). This clearly indicates that the uptake of insulin was enhanced when it was administered in the nanoparticulate form. The serum level of insulin after nasal administration of nanoparticles were found to be higher as compared to orally administered nanoparticles. Also, the serum insulin levels at the end of $2 \mathrm{~h}$ were found to be higher than our earlier reported results, where oral and nasal administration of insulin loaded sodium borohydride reduced gold nanoparticles lead to 1.7 and $1.2 \mathrm{mIU} / \mathrm{ml}$ insulin levels, respectively (11). The insulin levels determined after nasal administration of chitosan reduced gold nanoparticles $(2.1 \mathrm{mIU} / \mathrm{ml})$ is also comparable to that obtained after subcutaneous administration of insulin solution $(2.5 \mathrm{mIU} / \mathrm{ml})$, indicating that the nasal delivery of insulin by nanoparticles could be an alternative to painful and traumatic subcutaneous delivery in treatment of post prandial hyperglycemia. The uptake of chitosan reduced gold nanoparticles was investigated by determining the gold concentration in the serum using ICP measurement studies (Table IV). The serum gold levels for both oral and nasal administration of chitosan reduced gold nanoparticles were found to be in the range of $0.053-0.072 \mathrm{ppm}$. The serum gold levels were not significantly different after oral and nasal administration of insulin loaded chitosan reduced gold nanoparticles. However, efficacy after nasal administration was higher as compared to oral, indicative of some insulin degradation in the gastric environment. The serum gold levels following sodium borohydride reduced gold nanoparticles were obtained in the range of $0.021-0.032 \mathrm{ppm}$ (11). Thus, chitosan reduced gold nanoparticles have resulted in a significant improvement in the uptake. This provides sufficient evidence for our hypothesis of uptake enhancement property of chitosan reduced gold nanoparticles.

\section{CONCLUSIONS}

In conclusion, we have demonstrated a novel method for the synthesis of gold nanoparticles using a biocompatible polymer, chitosan with improved surface properties for binding of biomolecules. Also the uptake and penetration enhancement property of chitosan has been explored for administration of insulin by transmucosal route. Our studies indicate that oral and nasal administration of insulin loaded chitosan reduced gold nanoparticles has lead to improved pharmacodynamic activity as evidenced by higher reduction in BGL as compared to the insulin loaded sodium borohydride reduced gold nanoparticles. In the light of these results, chitosan reduced gold nanoparticles could be considered as a promising delivery system for transmucosal application of biologicals. The excretion, accumulation and toxicity of nanoparticles following chronic use are under investigation in our laboratory.

\section{ACKNOWLEDGEMENTS}

DRB is thankful to the Fair \& Lovely Foundation, for providing financial assistance in form of Project Saraswati Scholarship. The authors are thankful to Dr. Madhusudan Rao for providing the facility of zeta sizer.

\section{REFERENCES}

1. K. A. Janes, and M. J. Alonso. Depolymerised chitosan nanoparticles for protein delivery: preparation and characterization. J. Appl. Polym. Sci. 88:2769-2776 (2003). 
2. S. Shukla, A. Priscilla, M. Banerjee, R. R. Bhonde, J. Ghatak, P. V. Satyam, and M. Sastry. Porous gold nanospheres by controlled transmetallation reaction: a novel material for apllication in cell imaging. Chem. Mater. 17:5000-5005 (2005).

3. W. C. W. Chan, and S. Nie. Quantum dot bioconjugates for ultrasensitive non-isotopic detection. Science 281:2016-2018 (1998).

4. T. Jung, W. Kamm, A. Breitenbach, E. Kaiserling, J. X. Xiao, and T. Kissel. Biodegradable nanoparticles for oral delivery of peptides: is there a role for polymers to affect mucosal uptake? Eur. J. Pharm. Biopharm. 50:147-160 (2000).

5. H. Chen, and R. Langer. Oral particulate delivery: status and future trends. Adv. Drug Deliv. Rev. 34:339-350 (1998).

6. T. H. Ermak, and P. J. Giannasca. Microparticle targeting to M cells. Adv. Drug Del. Rev. 34:261-283 (1998).

7. G. A. Hughes. Nanostructure-mediated drug delivery. Nanomedicine: Nanotechnology, Biology, and Medicine 1:22-30 (2005).

8. H. Joshi, P. S. Shirude, V. Bansal, K. N. Ganesh, and M. Sastry. Isothermal titration calorimetry studies on the binding amino acid to gold nanoparticles. J. Phys. Chem., B. 108:11535-11540 (2004).

9. S. S. Shankar, A. Rai, A. Ahmad, and M. Sastry. Controlling the optical properties of lemongrass extract synthesized gold nanoparticles and potential application in infrared absorbing optical coatings. Chem. Mater. 17:566-572 (2005).

10. R. Shukla, V. Bansal, M. Chaudhary, A. Basu, R. Bhonde, and M. Sastry. Biocompatibility of gold nanoparticles and their endocytotic fate inside the cellular compartment: a microscopic overview. Langmuir 21:10644-10654 (2005).

11. H. M. Joshi, D. R. Bhumkar, K. Joshi, V. Pokharkar, and M. Sastry. Gold nanoparticles as carriers for efficient transmucosal insulin delivery. Langmuir 22:300-305 (2006).

12. A. P. Alivisatos, X. Peng, T. E. Wilson, C. L. Loweth, M. P. Bruchez Jr, and P. G. Schultz. Organization of nanocrystal molecules using DNA. Nature 382:609-611 (1996).

13. R. S. Ingram, M. J. Hostetler, and R. W. Murray. Poly-hetero- $\omega-$ functionalized alkanethiolate-stabilized gold cluster compounds. J. Am. Chem. Soc. 119:9175-9178 (1997).

14. C. M. Niemeyer. Nanoparticles, proteins, and nucleic acids: biotechnology meets materials science. Angew. Chem., Int. Ed. 40:4128-4158 (2001).

15. A. Bielinska, J. D. Eichman, I. Lee, J. R. Baker Jr, and L. Balogh. Imaging $\left\{\mathrm{Au}^{\circ}\right.$-PAMAM $\}$ gold-dendrimer nanocomposites in cells. J. Nanopart. Res. 4:395-403 (2002).

16. M. Thomas and M. A. Klibanov. Conjugation to gold nanoparticles enhances polyethylenimine's transfer of plasmid DNA into mammalian cells. Proc. Natl. Acad. Sci. U.S.A. 100:91389143 (2003).

17. L. Ren and G. M. Chow. Synthesis of nir-sensitive $A u-\mathrm{Au}_{2} \mathrm{~S}$ nanocolloids for drug delivery. Mater. Sci. Eng., C.23:113-116 (2003).

18. H. Gu, P. L. Ho, E. Tong, L. Wang, and B. Xu. Presenting vancomycin on nanoparticles to enhance antimicrobial activities. Nano Lett. 3:1261-1263 (2003).

19. L. R. Hirsch, R. J. Stafford, J. A. Bankson, S. R. Sershen, B. Rivera, R. E. Price, J. D. Hazle, N. J. Halas, and J. L. West. Nanoshell-mediated near-infrared thermal therapy of tumors under magnetic resonance guidance. Proc. Natl. Acad. Sci. U.S.A. 100:13549-13554 (2003).

20. M. C. Daniel, and D. Astruc. Gold nanoparticles: assembly, supramolecular chemistry, quantum-size-related properties, and applications toward biology, catalysis, and nanotechnology. Chem. Rev. 104:293-346 (2004).

21. M. Brust, M. Walker, D. Bethell, D. J. Schiffrin, and R. Whyman. Synthesis of thiol-derivatised gold nanoparticles in a two-phase liquid-liquid system. J. Chem. Soc., Chem. Commun. 7:801-802 (1994).

22. A. Silva-Cunha, M. Cheron, J. L. Grossiord, F. Puisieux, and M. Seiller. $\mathrm{W} / \mathrm{O} / \mathrm{W}$ multiple emulsions of insulin containing a protease inhibitor and an absorption enhancer: biological activity after oral administration to normal and diabetic rats. Int. J. Pharm. 169:33-44 (1998).

23. G. Rosa, R. Lommelli, M. L. La Rotonda, A. Miro, and F.
Quaglia. Influence of the co-encapsulation of different non-ionic surfactants on the properties of PLGA insulin-loaded microspheres. J. Control. Release 69:283-295 (2000).

24. E. A. Hosny, H. I. Al-Shora, and M. M. A. Elmazar. Oral delivery of insulin from enteric-coated capsules containing sodium salicylate: effect on relative hypoglycemia of diabetic beagle dogs. Int. J. Pharm. 237:71-76 (2002).

25. V. Agarwal, I. K. Reddy, and M. A. Khan. Polymethacrylate based microparticulates of insulin for oral delivery: preparation and in vitro dissolution stability in the presence of enzyme inhibitors. Int. J. Pharm. 225:31-39 (2001).

26. L. Illum. Chitosan and its use as a pharmaceutical excipient. Pharm. Res. 15:1326-1331 (1998).

27. O. Felt, P. Buri, and R. Gurny. Chitosan: a unique polysaccharide for drug delivery. Drug Dev. Ind. Pharm. 24:979-993 (1998).

28. Y. Pan, Y. Li, H. Zhao, J. Zheng, H. Xu, G. Wei, J. Hao, and F. Cui. Bioadhesive polysaccharide in protein delivery system: chitosan nanoparticles improve the intestinal absorption of insulin in vivo. Int. J. Pharm. 249:139-147 (2002).

29. Z. Ma, T. M. Lim, and L. Lim. Pharmacological activity of peroral chitosan-insulin nanoparticles in diabetic rats. Int. J. Pharm. 293:271-280 (2005)

30. I. M. V. Lubben, J. C. Verhoef, G. Borchard, and H. E. Junginger. Chitosan for mucosal vaccination. Adv. Drug Del. Rev. 52:139144 (2001).

31. E. Bjork, and P. Edman. Degradable starch microspheres as a nasal delivery system for insulin. Int. J. Pharm. 47:233-238 (1988).

32. I. M. V. Lubben, J. C. Verhoef, G. Borchard, and H. E. Junginger. Chitosan and its derivatives in mucosal drug and vaccine delivery. Eur. J. Pharm. Sci. 14:201-207 (2001).

33. A. Martin, J. Swarbrick, and A. Cammarata. Physical Pharmacy, Varghese, Mumbai, 1991.

34. I. Bertholon, G. Ponchel, D. Labarre, P. Couvreur, and C. Vauthier. Bioadhesive properties of poly (alkylcyanoacrylate) nanoparticles coated with polysaccharide. J. Nanosci. Nanotech. 6:3102-3109 (2006).

35. D. Cremaschi, S. Dossena, C. Porta, V. Rossi, and M. Pinza. Further analysis of transcytosis of free polypeptides and polypeptide-coated nanobeads in rabbit nasal mucosa. J. Appl. Physiol. 91:211-217 (2001)

36. R. Narayani. Oral delivery of insulin-making needles needless. Trends Biomater. Artif. Organs 15:12-16 (2001).

37. J. A. Galloway, C. T. Spradlin, R. L. Nelson, S. M. Wentworth, J. A. Davidson, and J. L. Swarner. Factors influencing the absorption, serum insulin concentration, and blood glucose responses after injections of regular insulin and various insulin mixtures. Diabetes Care 4:366-376 (1981).

38. F. A. Dorkoosh, J. C. Verhoef, G. Borchard, M. Rafiee-Tehrani, J. H. M. Verheijden, and H. E. Junginger. Intestinal absorption of human insulin in pigs using delivery systems based on superporous hydrogel polymers. Int. J. Pharm. 247:47-55 (2002).

39. A. M. Lowman, M. Morishita, M. Kajita, T. Nagai, and N. A. Peppas. Oral delivery of insulin using $\mathrm{pH}$-responsive complexation gels. J. Pharm. Sci. 88:933-937 (1999).

40. D. A. Norris, N. Puri, and P. J. Sinko. The effect of physical barriers and properties on the oral absorption of particulates. Adv. Drug Deliv. Rev. 34:135-154 (1998).

41. J. Blanchette, N. Kavimandan, and N. A. Peppas. Principles of transmucosal delivery of therapeutic agents. Biomed. Pharmacother. 58:142-151 (2004).

42. P. Yeh, H. Ellens, and P. L. Smith. Physiological considerations in the design of particulate dosage forms for oral vaccine delivery. Adv. Drug Deliv. Rev. 34:123-133 (1998).

43. L. Illum. Nasal drug delivery-possibilities, problems and solutions. J. Control. Release 87:187-198 (2003).

44. L. Illum. Transport of drugs from the nasal cavity to the central nervous system. Eur. J. Pharm. Sci. 11:1-18 (2000).

45. L. Illum. Nasal drug delivery: new developments and strategies. Drug Discov. Today 7:1184-1189 (2002). 
Reproduced with permission of the copyright owner. Further reproduction prohibited without permission. 